\title{
Structural Adaptations in Overwintering Leaves of Thermonastic and Nonthermonastic Rhododendron Species
}

\author{
Xiang Wang and Rajeev Arora ${ }^{1}$ \\ Department of Horticulture, Iowa State University, Ames, IA 50011-1100

\begin{abstract}
Harry T. Horner
Department of Genetics, Development and Cell Biology and Microscopy and NanoImaging Facility, Iowa State University, Ames, IA 50011-1020
\end{abstract}

\author{
Stephen L. Krebs \\ David G. Leach Research Station of the Holden Arboretum, Kirtland, OH 44094
}

AdDitional INDEX words. cold acclimation, leaf movement, photoinhibition, leaf anatomy

\begin{abstract}
Evergreen rhododendrons (Rhododendron L.) are important woody landscape plants in many temperate zones. During winters, leaves of these plants frequently are exposed to a combination of cold temperatures, high radiation, and reduced photosynthetic activity, conditions that render them vulnerable to photooxidative damage. In addition, these plants are shallow-rooted and thus susceptible to leaf desiccation when soils are frozen. In this study, the potential adaptive significance of leaf morphology and anatomy in two contrasting Rhododendron species was investigated. $R$. catawbiense Michx. (native to eastern United States) exhibits thermonasty (leaf drooping and curling at subfreezing temperatures) and is more winter-hardy [leaf freezing tolerance $\left(\mathrm{LT}_{\mathbf{5 0}}\right)$ of containerized plants $\approx-35$ ${ }^{\circ} \mathrm{C}$, whereas $R$. ponticum $\mathrm{L}$. (native to central Asia) is less hardy $\left(\mathrm{LT}_{\mathbf{5 0}} \approx-16{ }^{\circ} \mathrm{C}\right)$, and nonthermonastic. Thermonasty may function as a light and/or desiccation avoidance strategy in rhododendrons. Microscopic results revealed that $R$. ponticum has significantly thicker leaf blades but thinner cuticle than $R$. catawbiense. There is one layer of upper epidermis and three layers of palisade mesophyll in $R$. catawbiense compared with two distinct layers of upper epidermis and two layers of palisade mesophyll in $R$. ponticum. We suggest that the additional layer of upper epidermis in $R$. ponticum and thicker cuticle and extra palisade layer in $R$. catawbiense represent structural adaptations for reducing light injury in leaves and could serve a photoprotective function in winter when leaf photochemistry is generally sluggish. Results also indicate that although stomatal density of $R$. ponticum is higher than that of $\boldsymbol{R}$. catawbiense leaves, the overall opening of stomatal pores per unit leaf area (an integrated value of stomatal density and pore size) is higher by approximately twofold in $R$. catawbiense, suggesting that $R$. catawbiense may be more prone to winter desiccation and that thermonasty may be a particularly beneficial trait in this species by serving as a desiccation-avoidance strategy in addition to a photoprotection role.
\end{abstract}

Rhododendrons are among the most widely grown woody ornamentals in landscapes and public gardens with great horticultural interest. Rhododendron (Ericaceae) comprises almost 1000 species distributed worldwide (Chamberlain et al., 1996). Among them, over 800 species are distributed throughout the Northern Hemisphere, ranging from tropical to polar climates and varying widely in their cold-hardiness (Leach, 1961; Sakai et al., 1986). Overwintering perennials in the temperate zone survive through harsh winters by an induced process termed cold acclimation (CA). This seasonal phenomenon greatly increases their cold-hardiness in response to

Received for publication 11 July 2008. Accepted for publication 2 Oct. 2008. This journal paper of the Iowa Agriculture and Home Economics Experiment Station, Ames, Iowa (Project 3601) was supported by Hatch Act and State of Iowa funds.

We thank Tracey Pepper and Randall Den Adel of Microscopy and NanoImaging Facility (Department of Genetics, Development and Cell Biology, Iowa State University) for helpful assistance and advice for the microscopy work. We also thank Dr. Allen D. Knapp for providing the fluorometer and Ms. Maria M. Hartt (Department of Agronomy, Iowa State University) for technical advice and assistance with $\mathrm{F}_{\mathrm{v}} / \mathrm{F}_{\mathrm{m}}$ measurements. We thank Arlen Patrick (greenhouse manager, Department of Horticulture, Iowa State University) for assisting with plant care throughout this study.

${ }^{1}$ Corresponding author. E-mail: rarora@iastate.edu. inductive short photoperiods, low nonfreezing and then subfreezing temperatures sequentially through early fall and winter (Sakai and Larcher, 1987; Weiser, 1970). Evergreen rhododendrons are broad-leaved woody plants and the freezing tolerance of fully cold-acclimated leaves can be as low as -50 to $-60{ }^{\circ} \mathrm{C}$ (Sakai et al., 1986; Wei et al., 2005).

As understory evergreen plants in the deciduous forests, leaves of most rhododendrons are commonly exposed to a combination of freezing temperatures and high light in their natural habitat during winter. A previous study in our laboratory indicated that downregulation of photosynthetic metabolism [specifically five photosynthesis-related genes (light-harvesting chlorophyll a/b-binding protein, $\mathrm{RuBisCO}$ small subunit precursor, RuBisCO activase, plastidic fructose bisphosphate aldolase, and chloroplast precursor of plastocyanin)] in overwintering leaves of $R$. catawbiense (Wei et al., 2005) could potentially result in light energy harvested by the leaves to be in excess of what can be processed by photosystems, thus making these plants particularly vulnerable to photoinhibition or photooxidative damage (Peng et al., 2008).

Many plants have evolved mechanisms to dissipate excess absorbed light safely as thermal energy through the xanthophyll cycle (Adams et al., 2004) and/or protect cells against the 
photo-oxidative damage through antioxidants such as tocopherols, ascorbate, and glutathione (Niyogi, 1999). Recent studies with several overwintering evergreens-rhododendron $(R$. catawbiense), subalpine fir [Abies lasiocarpa (Hook.) Nutt.], and lodgepole pine (Pinus contorta Dougl. ex Loud.)-showed that the xanthophyll pool was significantly upregulated in sunexposed winter leaves compared with the summer leaves and that it may play a key role in the prevention of photo-oxidative damage (Harris et al., 2006; Zarter et al., 2006a, 2006b).

The leaves of some evergreen Rhododendron species show thermonasty (i.e., temperature-induced leaf movements), a phenomenon in which leaves droop and curl at freezing temperatures in winter (Nilsen, 1987). Thermonasty or thermonastylike responses have been observed on main stems of Phryma leptostachya L. (Endo and Miyauchi, 2006) and the petals of some flowers (Tulipa L. and Crocus L.) (Crombie, 1962); however, these movements occur at relatively warmer temperatures $\left(\approx 12{ }^{\circ} \mathrm{C}\right.$ for $P$. leptostachya $)$, whereas leaf thermonasty in rhododendrons typically occurs at subfreezing temperatures (Nilsen, 1987). Nilsen (1992) proposed several possible theories for thermonasty response in rhododendrons. A hypothesized adaptive benefit of thermonastic leaf movement is avoidance of high light stress by reducing the leaf area and shading the leaf more. It has been suggested that without thermonasty, the leaves would be injured during bright, cold winter days and, as a consequence, the net photosynthesis rate in the next year would decrease and leaf mortality would increase (Bao and Nilsen, 1988). Another possible adaptive benefit of thermonasty is prevention of desiccation during cold periods (Nilsen, 1992). Rhododendrons are typically shallowrooted plants and thus are susceptible to leaf desiccation under windy and/or freezing conditions in winter. Leaf curling could reduce transpiration by creating more humid microsites around the stomata of the lower leaf surface (Nilsen, 1992).

In addition to light avoidance and tolerance mechanisms, many plants exhibit photoacclimation in response to light stress that is manifested in the leaf morphological and anatomical adaptations (Anderson et al., 1995; Björkman, 1981; Walters et al., 2003). Photoacclimation can occur at two levels: 1) leaf level and 2) chloroplast level (Murchie and Horton, 1997). The different anatomy of "sun" and "shade" leaves is an example of leaf level acclimation (Björkman, 1981). For example, "sun" leaves are smaller and thicker with more columnar mesophyll cells than "shade" leaves and have a higher density of leaf stomata (Lichtenthaler et al., 1981; Nobel, 1977; Oguchi et al., 2003; Sims and Pearcy, 1992; Wentworth et al., 2006) as well as increased lengths of stomatal pores (Wild and Wolf, 1980). Because the leaves of overwintering rhododendrons are typically exposed to excess light in winter, they too are expected to have evolved leaf morphological and anatomical features to allow them to handle the high irradiance.

The objective of this study was to compare the leaf structural differences of the two Rhododendron species ( $R$. catawbiense and $R$. ponticum) that are divergent in their leaf freezing tolerance (superhardy versus less hardy, respectively) and in their thermonastic behavior (former shows thermonasty, whereas the latter does not). We hope to gain insight into how thermonasty (or lack thereof) together with the specific leaf morphological and anatomical features of the two species might be associated with their ability to tolerate/avoid high irradiance and/or withstand dehydrative stresses during winter.

\section{Materials and Methods}

Plant material and growth Conditions. The plants from the two species of Rhododendron [R. catawbiense 'Catalgla' and $R$. ponticum 'RSBG 76/411' (RSBG = Rhododendron Species Foundation and Botanical Garden)] were vegetatively propagated (semihardwood cuttings) and grown in 19-L plastic pots at the David G. Leach Research Station of Holden Arboretum (Kirtland, OH). Approximately 2-year-old containerized plants were sent to the Department of Horticulture at Iowa State University (ISU) in Ames, where they were maintained in Fafard mix 52 (Conrad Fafard, Agawam, MA) [pine bark (60\%); peat, perlite, and vermiculite (40\%)] outside the ISU horticulture greenhouse (lat. $42^{\circ} 1^{\prime} \mathrm{N}$, long. 93 $38^{\prime} \mathrm{W}$ ). The plants were allowed to acclimate naturally from Aug. to Dec. 2007. Average monthly minimum air temperatures from Aug. to Dec. 2007 at this location were 16.9, 11.1, 7.7, -2.8, and $-10.8{ }^{\circ} \mathrm{C}$, respectively. All the plants were partly shaded by placing the pots in the understory of a pine (Pinus L.) tree. Daily changes of irradiance on leaf surface on sunny days were quite stable and the irradiance ranged from $75 \mu \mathrm{mol} \cdot \mathrm{m}^{-2} \cdot \mathrm{s}^{-1}(0800 \mathrm{HR})$ to $1300 \mu \mathrm{mol} \cdot \mathrm{m}^{-2} \cdot \mathrm{s}^{-1}(1400 \mathrm{HR})$ with an average of $\approx 350$ $\mu \mathrm{mol} \cdot \mathrm{m}^{-2} \cdot \mathrm{s}^{-1}$. The plants were watered as needed throughout the study.

Freezing tolerance tests. Leaf freezing tolerance (FT) tests were performed by subjecting punched leaf discs (diameter $=1.27 \mathrm{~cm}$ ) to a controlled freeze-thaw regime followed by the assessment of injury by measuring electrolyte leakage from freeze-thaw-injured tissues as described by Lim et al. (1998). The discs were cooled in a temperature-controlled glycol bath (Isotemp 3028; Fisher Scientific, Pittsburgh, PA) for nonacclimated (NA) leaves sampled in Aug. 2007 or in a programmable freezer (model 85-3.1; ScienTemp, Adrian, MI) for CA leaves sampled in Dec. 2007. The lowest treatment temperatures for NA samples (in the glycol bath) and CA samples (in the programmable freezer) were -20 and $-50{ }^{\circ} \mathrm{C}$, respectively. Two separate cooling devices were used in this study because the former's cooling limit is only up to $-22{ }^{\circ} \mathrm{C}$; however, ice nucleation (at $\approx-1$ and $-3{ }^{\circ} \mathrm{C}$ for the glycol bath and programmable freezer, respectively) was ensured in all the freezing tests and tissues were cooled at relatively slow cooling rates $\left(-1\right.$ to $\left.-2{ }^{\circ} \mathrm{C} / \mathrm{h}\right)$. The treatment temperatures at which NA or CA samples were removed from the cooling bath or the programmable freezer were at -2 to -3 and $-5{ }^{\circ} \mathrm{C}$ increments, respectively, and included an unfrozen control. Fully expanded leaves from current-year growth were collected to conduct the tests. Three replications were made at each treatment temperature from three individual leaves (one leaf from each of three plants per species).

For NA leaves, punched leaf discs were placed in test tubes with $80 \mu \mathrm{L}$ demineralized water; the tubes were placed in the glycol bath for $1 \mathrm{~h}$ at $-1{ }^{\circ} \mathrm{C}$ and ice chips were added to initiate ice nucleation. After an additional hour at $-1{ }^{\circ} \mathrm{C}$, the temperature was cooled at a rate of $1{ }^{\circ} \mathrm{C}$ per hour. Frozen samples at each treatment temperature were removed from the glycol bath and thawed on ice overnight. Samples were removed from ice and thawed at $4{ }^{\circ} \mathrm{C}$ for $1 \mathrm{~h}$ and then at room temperature for another hour. Ions were extracted with $20 \mathrm{~mL}$ demineralized water followed by vacuum infiltration (three times for $3 \mathrm{~min}$ each at $\approx 100 \mathrm{kPa}$ ) and shaking for $3 \mathrm{~h}$ at $250 \mathrm{rpm}$. Initial electrical conductivity was measured for each sample with a conductivity meter (model 3100; YSI, 
Yellow Spring, OH). Final electrolyte leakage for each sample was determined after autoclaving the samples at $121{ }^{\circ} \mathrm{C}$ for $20 \mathrm{~min}$. Percent ion leakage at each temperature was calculated and converted to percentage injury, and then $\mathrm{LT}_{50}$ (the temperature at which 50\% injury occurred and defined as FT) was calculated according to Lim et al. (1998). For cold-acclimated samples, leaf discs were cooled in the programmable freezer with ice nucleation occurring at $\approx-2$ to $-3{ }^{\circ} \mathrm{C}$ with the cooling rate of $\approx 1{ }^{\circ} \mathrm{C}$ per hour during the first $4 \mathrm{~h}$ (up to $-3{ }^{\circ} \mathrm{C}$ ) and $\approx 4{ }^{\circ} \mathrm{C}$ per hour until the lowest treatment temperature was reached. All the remaining processes were the same as those conducted for NA samples.

Chlorophyll fluorescence measurements. Modulated chlorophyll fluorescence was measured with a fluorometer (PAM-2000; Waltz, Effetrich, Germany) and analyzed by Data Acquisition Software DA-2000 (Waltz). Both NA (August) and CA (November) current-year leaves from the two species were used. Intact leaves were dark-adapted for $30 \mathrm{~min}$ to measure the

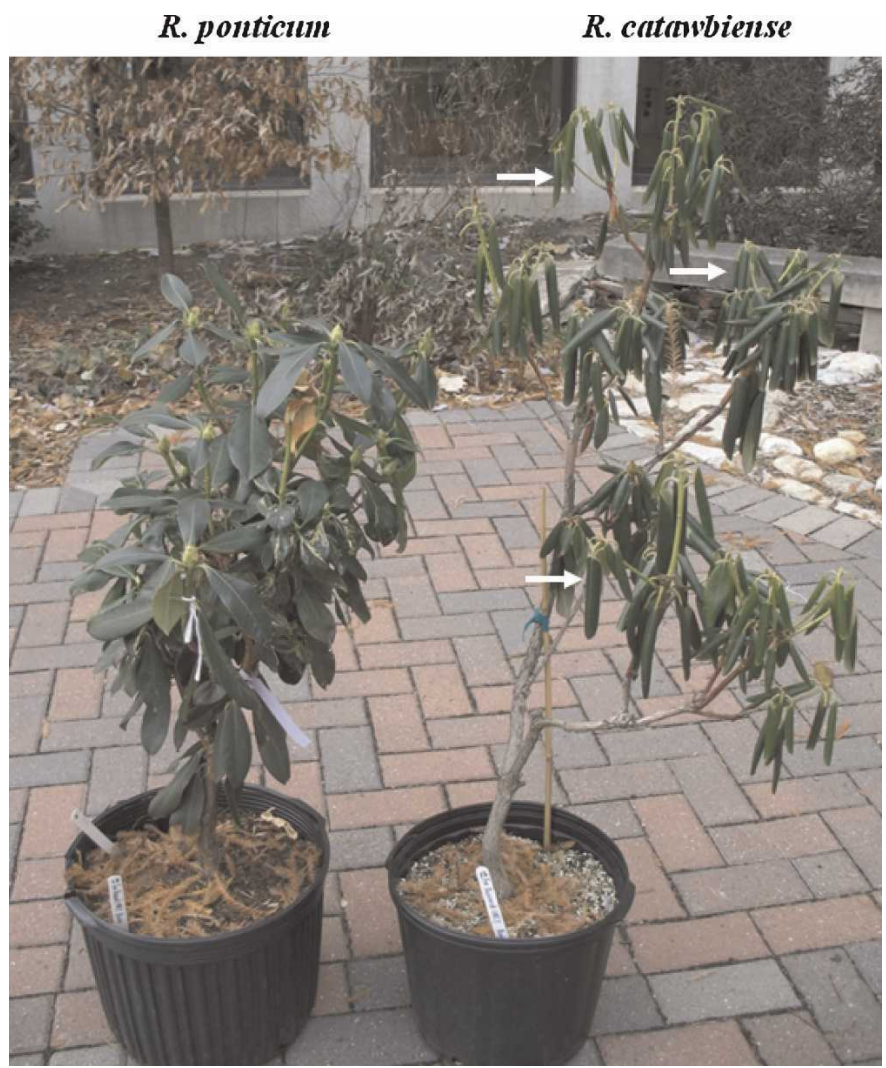

Fig. 1. The photograph showing no leaf movement and thermonastic leaf movement (see arrows) in Rhododendron ponticum and $R$. catawbiense, respectively. The picture was taken on a sunny day while the ambient temperature was $-5.5^{\circ} \mathrm{C}$ in Dec. 2006 . maximal quantum yield of photosystem II $\left(\mathrm{F}_{\mathrm{v}} / \mathrm{F}_{\mathrm{m}}\right)$ according to Heddad et al. (2006). This parameter reflects the maximal efficiency of photosystem II that is measured in dark-adapted tissues. Three leaves from three different plants were used for all measurements (one leaf from each of the three plants per species). All measurements were made between 1000 and 1200 HR on sunny days.

Light AND SCANNing ELECTRON Microscopy. Punches (diameter $=1 \mathrm{~mm}$ ) were made from fully expanded leaves at room temperature (NA leaves) or in the cold room (CA leaves) to be used for microscopy analysis. Punches from three separate leaves (one leaf from each of three plants/species and several punches/leaf) were made from the midlength along midribs and three punches/species (one punch/leaf) were randomly selected for microscopy.

For light microscopy (LM), leaf discs were fixed in $2 \%$ paraformaldehyde $/ 2 \%$ glutaraldehyde in $0.1 \mathrm{M}$ cacodylate buffer ( $\mathrm{pH}$ 7.2) under low vacuum for at least $2 \mathrm{~h}$ and then stored at $4{ }^{\circ} \mathrm{C}$ overnight. The fixed tissues were washed in the cacodylate buffer, dehydrated in a graded ethanol series $(25 \%$, $50 \%, 70 \%, 95 \%, 100 \%$ ), and then transferred to $100 \%$ acetone. Each wash lasted $30 \mathrm{~min}$. The dehydrated discs were gradually infiltrated with a low-viscosity epoxy resin (Spurr, 1969) and embedded and polymerized at $60{ }^{\circ} \mathrm{C}$ for $48 \mathrm{~h}$. Three thin transverse sections $(0.5 \mu \mathrm{m})$ from each species were made with an ultramicrotome using glass knives, mounted on microscope slides, and stained with toluidine blue $\mathrm{O}$. The LM images were taken on a light microscope (BH10; Olympus, Center Valley, PA) with bright-field optics.

For scanning electron microscopy (SEM), the tissues fixed for LM study were postfixed with $1 \%$ osmium tetroxide $\left(\mathrm{OsO}_{4}\right)$, dehydrated in a graded ethanol series, and critical point dried using liquid $\mathrm{CO}_{2}$ (DCP-1; Denton Vacuum, Moorestown, NJ). Samples were then attached to aluminium stubs using adhesive tabs and silver painted around their edges. The mounted samples were sputter-coated with gold/palladium (20:80) and viewed with a JEOL JSM-5800 LV scanning electron microscope (JEOL, Tokyo, Japan) at $10 \mathrm{kV}$. Images were digitally captured.

All anatomical data were obtained using SEM images from NA and CA samples with at least three replications. These parameters included thickness of leaf blades and adaxial epidermis, depth of palisade parenchyma, thickness of leaf adaxial cuticle, stomatal density, and length and width of stomatal pores. Total opening area of stomatal pores per unit leaf area was calculated by integrating stomatal density (number of stomata/unit leaf area) and average stomatal pore size (width and length).

Statistical analysis. Statistical significance of differences between the two species in measured parameters was tested by Student's $t$ test. Means were considered to be significantly different when $P \leq 0.05$.

Table 1. Leaf freezing tolerance $\left(\mathrm{LT}_{50}\right)$ and maximal quantum yield of photosystem II $\left(\mathrm{F}_{\mathrm{v}} / \mathrm{F}_{\mathrm{m}}\right)$ of Rhododendron ponticum and R. catawbiense from nonacclimated (NA) and cold-acclimated (CA) leaves in $2007 .{ }^{z}$

\begin{tabular}{lcccc}
\hline & \multicolumn{2}{c}{$\mathrm{LT}_{50}\left({ }^{\circ} \mathrm{C}\right)$} & \multicolumn{2}{c}{$\mathrm{F}_{\mathrm{v}} / \mathrm{F}_{\mathrm{m}}$} \\
\cline { 2 - 5 } Species & NA (August) & CA (December) & NA (August) & $0.76 \mathrm{a} \pm 0.01$ \\
\hline R. ponticum & $-4.7 \mathrm{a} \pm 0.2$ & $-15.6 \mathrm{c} \pm 1.0$ & $0.66 \mathrm{~b} \pm 0.01$ \\
R. catawbiense & $-7.1 \mathrm{~b} \pm 0.4$ & $-34.5 \mathrm{~d} \pm 1.8$ & $0.74 \mathrm{a} \pm 0.02$ & $0.59 \mathrm{c} \pm 0.03$ \\
\hline
\end{tabular}

${ }^{\mathrm{z}}$ Data presented are mean \pm SE $(\mathrm{n}=3)$. Different letters indicate significant differences between species and sampling dates $(P \leq 0.05)$. 


\section{R ponticum}

Le

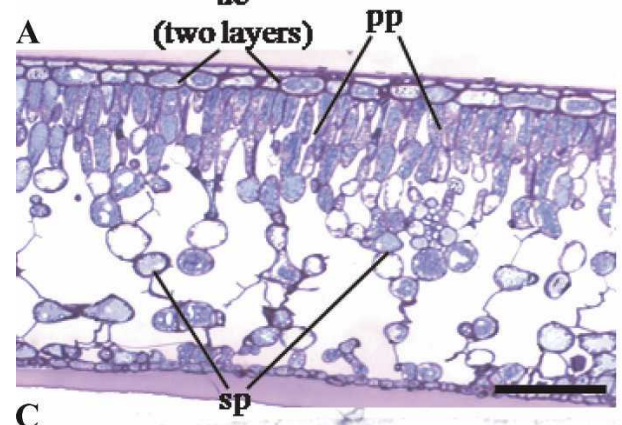

C

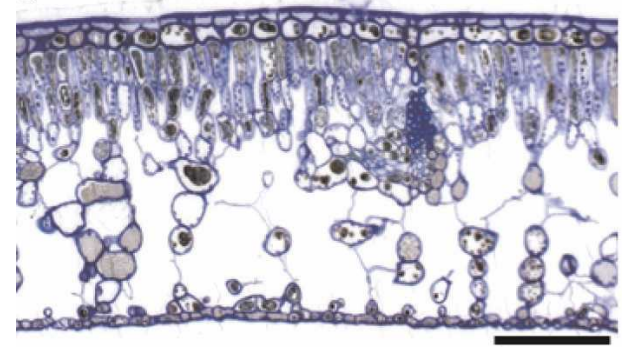

R. catawbiense

ue

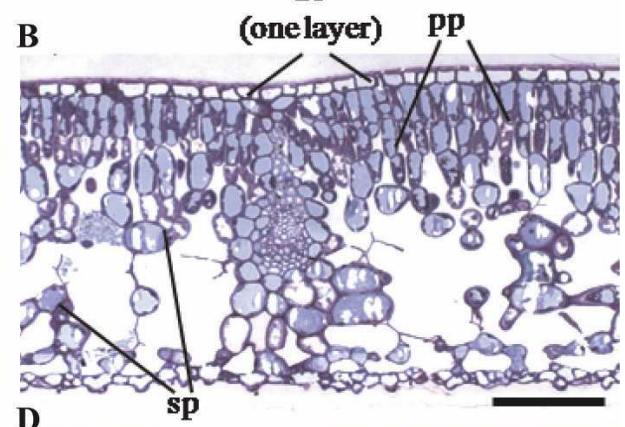

D

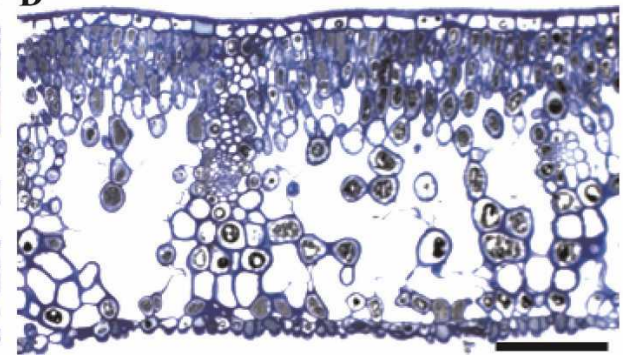

Fig. 2. Light micrographs of laminal anatomy of nonacclimated (NA) (A-B) and cold-acclimated (CA) (C-D) leaves from Rhododendron ponticum and $R$. catawbiense. (A, C) Transverse sections of NA and CA leaves from $R$. ponticum, respectively. (B, D) Transverse sections of NA and CA leaves from $R$. catawbiense, respectively. Bars $=100 \mu \mathrm{m} . \mathrm{pp}=$ palisade parenchyma, $\mathrm{sp}=$ spongy parenchyma, $\mathrm{ue}=$ upper (adaxial) epidermis.
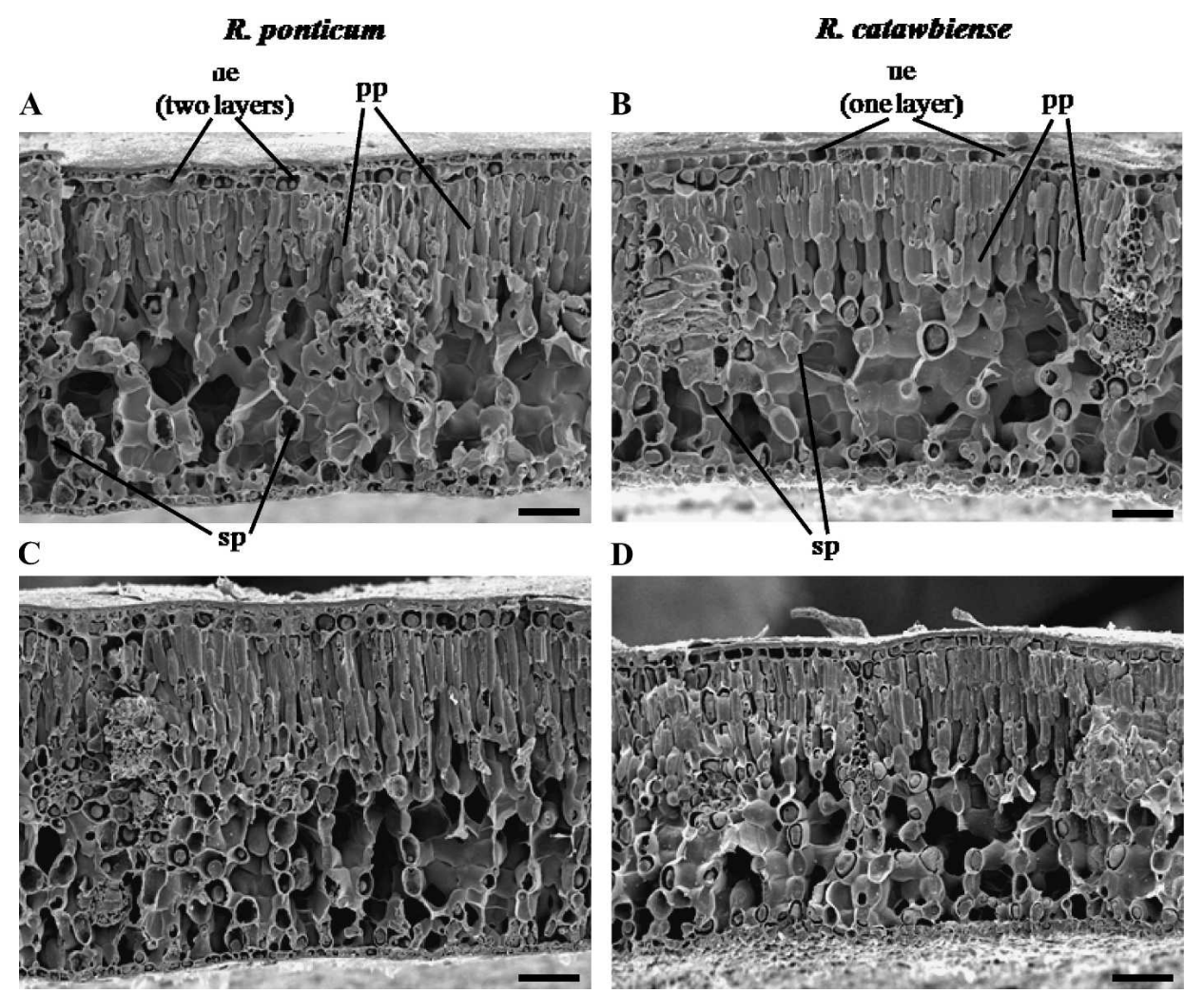

Fig. 3. Scanning electron micrographs of laminal anatomy of nonacclimated (NA) (A-B) and cold-acclimated (CA) (C-D) leaves from Rhododendron ponticum and $R$. catawbiense. (A, C) Leaf-fracture images of NA and CA plants from $R$. ponticum, respectively. Bars $=100 \mu \mathrm{m}$. (B, D) Leaf-fracture images of NA and CA plants from $R$. catawbiense, respectively. Bars $=100 \mu \mathrm{m} . \mathrm{pp}=$ palisade parenchyma, $\mathrm{sp}=$ spongy parenchyma, ue $=$ upper (adaxial) epidermis.

\section{Results}

Thermonasty, leaf freezing

tolerance, and maximal quantum

yield of photosystem II $\left(\mathbf{F}_{\mathrm{v}} / \mathbf{F}_{\mathrm{m}}\right)$

Thermonastic leaf movement at subfreezing temperatures $\left(-5.5^{\circ} \mathrm{C}\right)$ clearly appeared in CA leaves of $R$. catawbiense, but not in $R$. ponticum (Fig. 1). Leaf freezing tolerance $\left(\mathrm{LT}_{50}\right)$ of $R$. ponticum and $R$. catawbiense was -4.7 and $-7.1{ }^{\circ} \mathrm{C}$ for NA plants and -15.6 and $-34.5{ }^{\circ} \mathrm{C}$ for CA plants, respectively. Compared with $R$. ponticum, the $\mathrm{LT}_{50}$ of R. catawbiense was $58 \%$ and $121 \%$ lower (more negative) in NA and CA plants, respectively. Both species showed photoinhibition in November (lower values of $\mathrm{F}_{\mathrm{v}} / \mathrm{F}_{\mathrm{m}}$ ) compared with the measurements in August (Table 1). R. catawbiense showed greater photoinhibition than $R$. ponticum, especially in CA leaves $(20 \%$ versus $13 \%$ reduction in $\mathrm{F}_{\mathrm{v}} / \mathrm{F}_{\mathrm{m}}$; Table 1$)$.

Leaf anatomy of $R$. ponticum and R. catawbiense

LeAF BLAdes. LM and SEM images of anatomical features of NA and CA leaf blades are shown with Figures 2 and 3. There were two distinct layers of upper (adaxial) epidermis and two to three layers of palisade mesophyll in $R$. ponticum (Figs. 2A, 2C, 3A, and $3 \mathrm{C}$ ); however, there was one layer of adaxial epidermis and three to four layers of palisade mesophyll in $R$. catawbiense (Figs. 2B, 2D, 3B, and $3 \mathrm{D})$.

Thickness of leaf blades of $R$. ponticum was greater than that of $R$. catawbiense in both NA and CA leaves (Fig. 4A). Moreover, compared with NA leaves, thickness of leaf blades from CA plants increased significantly (by $\approx 17 \%$ ) in $R$. ponticum; however, no such change was observed in $R$. catawbiense. Thickness of adaxial epidermis of $R$. ponticum was also greater by approximately twofold than that of $R$. catawbiense in both NA $(\approx 25.7$ versus $\approx 14.6 \mu \mathrm{m})$ and $\mathrm{CA}(\approx 28.6$ versus $\approx 14.6 \mu \mathrm{m})$ leaves (Fig. 4B). Compared with NA leaves, thickness of adaxial epidermis from CA plants was significantly greater (by $\approx 12 \%$ ) in $R$. ponticum; however, no such change 

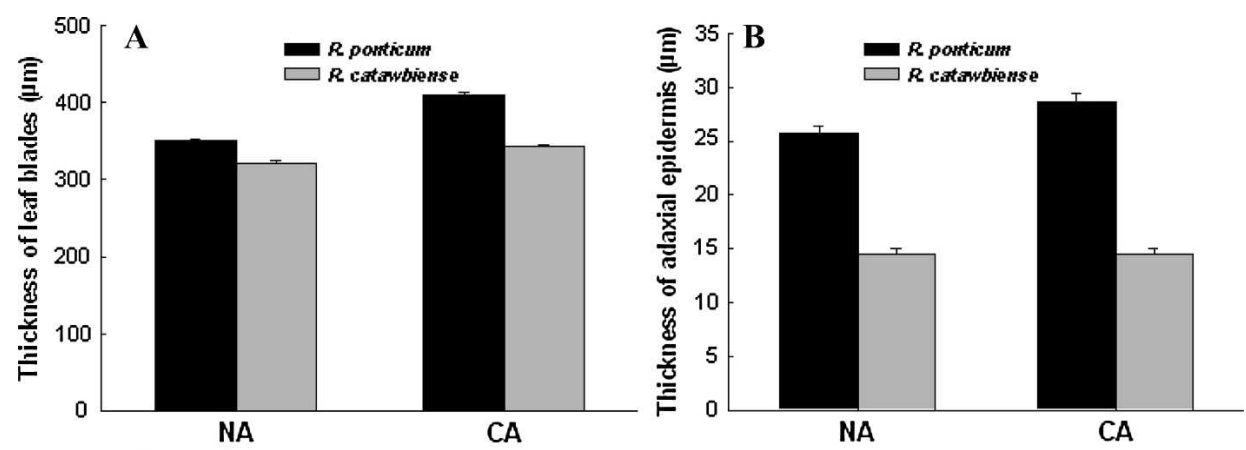

of $R$. ponticum $(\approx 17,970$ versus $\approx 6,081 \mu \mathrm{m}^{2} / \mathrm{mm}^{2}$ ) in NA leaves, whereas it was 1.9 -fold $(\approx 11,807$ versus $\approx 6,277 \mu \mathrm{m}^{2} / \mathrm{mm}^{2}$ ) (Fig. $7 \mathrm{G}$ ) that of $R$. ponticum in CA leaves.

\section{Discussion}

The relationships among thermonastic leaf movements, leaf freezing tolerance, and photoinhibition $\left(\mathrm{F}_{\mathrm{v}} / \mathrm{F}_{\mathrm{m}}\right)$ in rhododendron are not well understood. Our results showed that the thermonastic species ( $R$. catawbiense) (Fig. 1) had higher leaf freezing tolerance than the nonthermonastic species $(R$. ponticum) in both NA and CA leaves (Table 1). A previous study of 32 Rhododendron species exhibited a strong positive correlation between the degree of leaf curling and freezing tolerance (Nilsen and Tolbert, 1993), suggesting that thermonastic leaf movements may play a functional role in freezing tolerance in some Rhododendron species.

Our results showed that $R$. cata-

was observed in $R$. catawbiense. Depth of the palisade parenchyma of $R$. ponticum was relatively smaller (by $\approx 14 \%$ ) than that of $R$. catawbiense in NA leaves, but somewhat greater (by $\approx 4 \%$ ) than that of $R$. catawbiense in CA leaves (Fig. $4 C)$. Consequently, depth of the palisade parenchyma in CA plants increased $\approx 26 \%$ compared with NA leaves in $R$. ponticum; however, there was no change in $R$. catawbiense (Fig. 4C).

Thickness of adaxial cuticle from NA and CA leaves is shown in Figure 5A-E. Compared with $R$. ponticum, cuticle thickness in $R$. catawbiense was $\approx 16 \%$ and $\approx 7 \%$ greater in NA (5.1 versus $4.4 \mu \mathrm{m})$ and $\mathrm{CA}(6.3$ versus $5.9 \mu \mathrm{m})$ leaves, respectively (Fig. 5E). Also, CA leaves exhibited significantly greater cuticle thickness than NA leaves in both species with an increase of $\approx 34 \%$ and $\approx 24 \%$ for $R$. ponticum and $R$. catawbiense, respectively (Fig. 5E).

DiFFERENCES IN ABAXIAL STOMATA PROPERTIES. No wax could be observed around the stomata in $R$. ponticum (Figs. 6A and 6C), whereas a substantial amount of wax was noted in $R$. catawbiense (Figs. 6B and 6D). Stomatal density of $R$. ponticum was significantly higher than that of $R$. catawbiense in both NA $\left(\approx 335\right.$ versus $\approx 222$ stomata $\left./ \mathrm{mm}^{-2}\right)$ and CA $(\approx 306$ versus $\approx 128$ stomata $/ \mathrm{mm}^{-2}$ ) leaves (Fig. 6E). Compared with NA leaves, both species showed a significant decrease in stomatal density in CA leaves by $\approx 41.9 \%$ and $\approx 8.6 \%$ for $R$. catawbiense and $R$. ponticum, respectively (Fig. 6E). The two species showed different size (length, width, and opening area of pores) and shape of stomatal pores (Figs. 7A-D). Compared with $R$. ponticum, $R$. catawbiense showed significantly greater values (averaged over NA and CA samples) for the length $(\approx 13.4$ versus $\approx 7.1 \mu \mathrm{m})$ and width $(\approx 6.3$ versus $\approx 2.7 \mu \mathrm{m})$ (Figs. 7E and 7F, respectively). The opening area of stomatal pores/leaf area for $R$. catawbiense leaves was 2.9-fold that wbiense leaves were more photoinhibited than $R$. ponticum leaves in both NA and CA samples, but particularly more pronounced in winter (Table 1), indicating that $R$. catawbiense is more photosensitive than $R$. ponticum under comparable conditions. It is known that rhododendron plants, most of which belong to shade-loving species, experience the largest radiation of the year in winter because irradiance under a canopy of deciduous trees is higher in winter than in the summer. Thermonastic leaf movements of rhododendron plants at subfreezing temperatures were thought to alleviate photoinhibition (Nilsen, 1992) by reducing the quantity of light impinging on the leaf, thereby preventing or limiting photoinhibition. Thus, we suggest that leaf movements in $R$. catawbiense together with other leaf adaptations may help reduce photoinhibition in winter in this species, which often grows in the open (e.g., the so-called "balds" above the tree line in the Appalachian Mountains) and, hence, is more prone to light stress. The nonthermonastic species ( $R$. ponticum), however, may have evolved other avoidance or tolerance mechanisms for maintaining photosystem II photochemical efficiency under winter conditions.

Significantly different anatomy between "sun" and "shade" leaves is a good example of photoacclimation (Björkman, 1981). Our results showed that both species had some characteristics of "sun" leaves. For example, R. ponticum had thicker leaf blades (Fig. 4A) and higher density of leaf stomata (Fig. $6 \mathrm{E})$, the characteristics typically observed in "sun" leaves (Oguchi et al., 2003; Sims and Pearcy, 1992; Wentworth et al., 2006). Two-layered adaxial epidermis in $R$. ponticum leaves is a rather special feature and contributes to thicker leaf blades in this species. Our data indicated that, in this species, leaf thickness further increased in cold-acclimated tissues. Similar cold acclimation-induced leaf thickness has been previously 
A

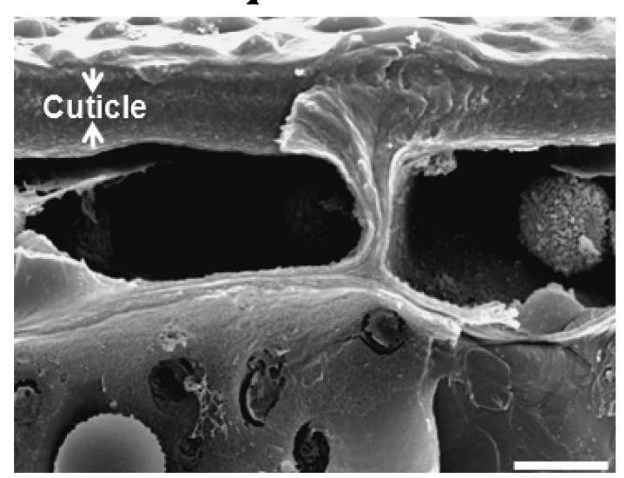

C
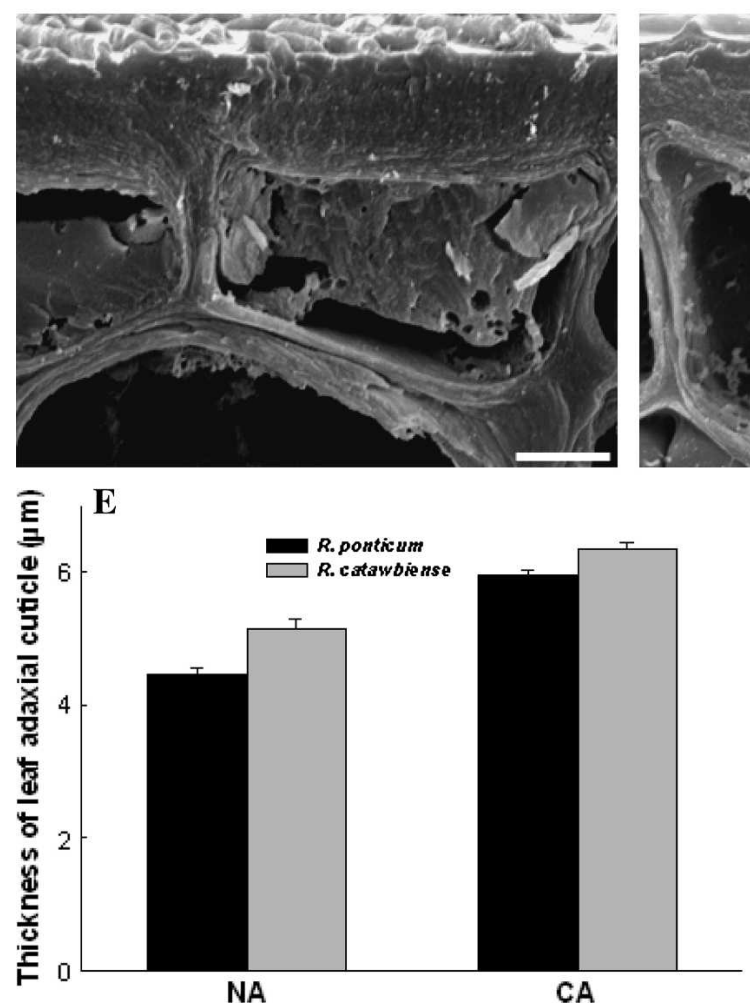

Fig. 5. Scanning electron micrographs showing thickness of adaxial cuticles of nonacclimated (NA) (A-B) and cold-acclimated (CA) (C-D) leaves from Rhododendron ponticum and $R$. catawbiense. $(\mathbf{A}, \mathbf{C})$ Images showing cuticle thickness of NA and CA leaves from $R$. ponticum, respectively. Bars $=5 \mu \mathrm{m}$. (B, D) Images showing cuticle thickness of NA and CA plants from $R$. catawbiense, respectively. Bars $=5 \mu \mathrm{m}$. (E) Comparison of thickness of adaxial cuticle from NA and CA leaves of $R$. ponticum and $R$. catawbiense. Note that cuticle thickness is marked by arrows. Data presented are mean $\pm \mathrm{SE}(\mathrm{n}=10$ independent measurements from three sections per species; $3+3+4$ ). Leaf samples were fixed and treated the same way as described in Fig. 3 .

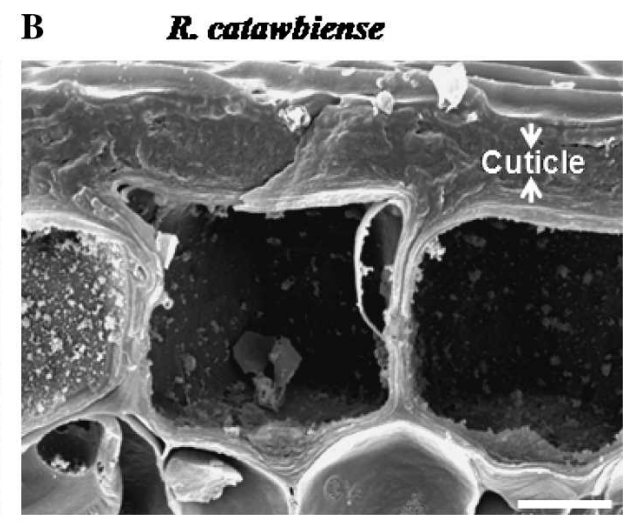

D

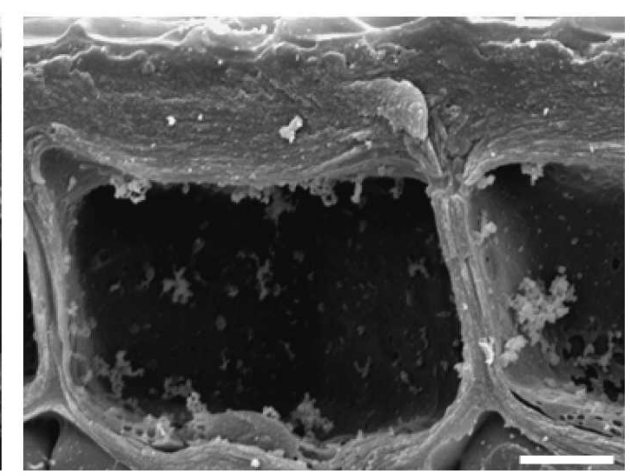

$$
\text { . }
$$
movement differences between these two species indicate that both species have evolved different mechanisms or adaptive structures to tolerate/avoid high irradiance in winter. However, data from future studies on potential differences in the light absorptivity and lightinduced injury in these two species should be useful in supporting this observation.

Cuticles, as the primary interface between the plant and its environment, receive a significant amount of solar radiation (Osborn and Taylor, 1990). They play a key role in maintaining the plant's integrity within an inherently hostile environment and provide a protective barrier to unfavorable light or water. Typically, cuticles of "sun" leaves are thicker (Martin and Juniper, 1970). Also, an increase in accumu-

reported in other species such as rye and winter oilseed rape and attributed to either increased mesophyll cell size or cell wall thickness (Huner et al., 1981; Stefanowska et al., 1999). Leaf epidermal cells are thought to protect leaves against high irradiance (Martin and Juniper, 1970) and/or ultraviolet radiation (Liakoura et al., 2003). The additional layer of upper epidermis and thicker adaxial epidermal cells in $R$. ponticum (Figs. 2A, 2C, and 4B) could be structural adaptations presumably associated with the photoprotection (from total light and/or specifically ultraviolet) during winter in this species.

Our results also showed that although $R$. catawbiense leaves had only one layer of upper epidermis, they contained an extra lating cuticle wax is a response of many plants to higher irradiance (Reed and Tukey, 1982; Shepherd et al., 1995) and cuticle wax is considered a photoprotective layer (Shepherd and Griffiths, 2006) by reflecting and diffusing light. In our study, $R$. catawbiense had thicker cuticles (Fig. 5) with a large accumulation of cuticle wax (Fig. 6) on the surface of the abaxial epidermis cells compared with $R$. ponticum in both NA and CA leaves, rendering $R$. catawbiense leaves to be more like "sun" leaves. Thus, we suggest that the thicker cuticles together with the accumulation of cuticle wax in the leaves of $R$. catawbiense may have adaptive significance vis-a-vis photoprotection in this species. 
A

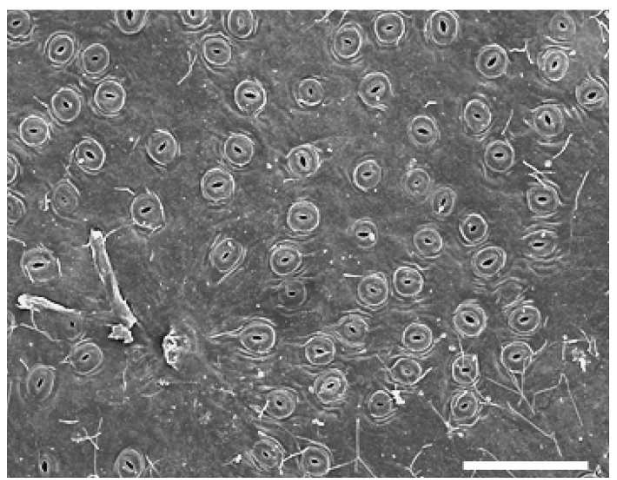

C
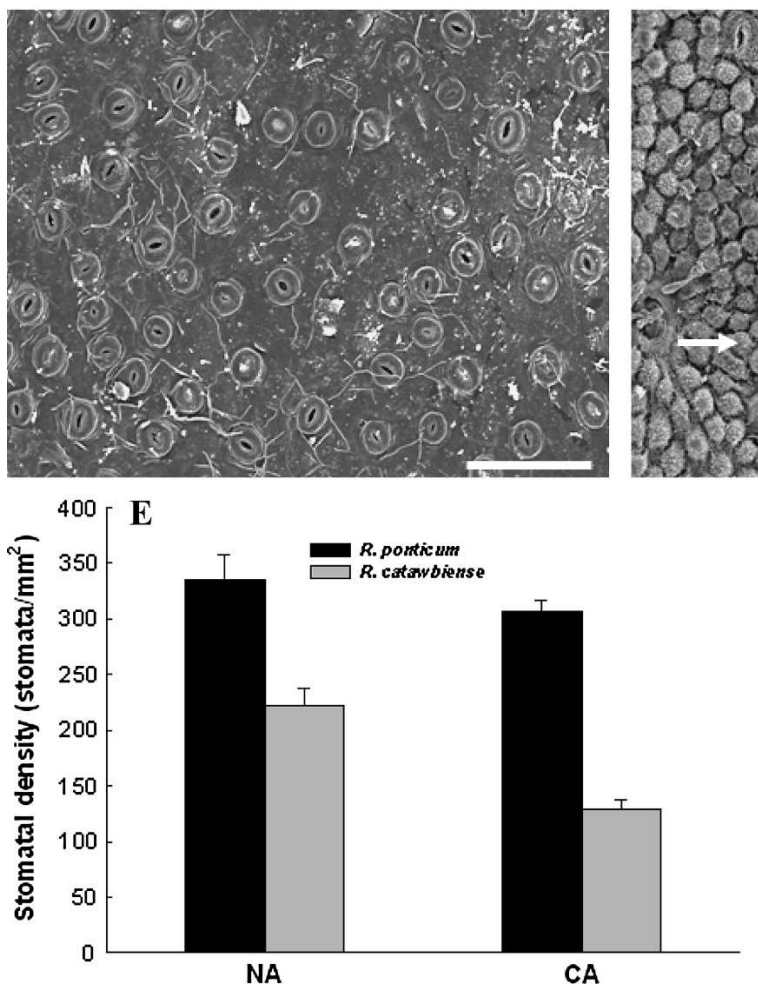

Fig. 6. Scanning electron micrographs showing the abaxial stomatal density of nonacclimated (NA) (A-B) and cold-acclimated (CA) (C-D) leaves from Rhododendron ponticum and $R$. catawbiense. (A, C) Images showing stomatal density of NA and CA leaves from $R$. ponticum, respectively. Note that there was no wax accumulated around the stomata. Bars $=100 \mu \mathrm{m}$. $(\mathbf{B}, \mathbf{D})$ Images showing stomatal density of NA and CA plants from $R$. catawbiense, respectively. Note the wax accumulated around the stomata (arrows). Bars $=100 \mu \mathrm{m}$. (E) Comparison of stomata density from NA and CA leaves of $R$. ponticum and $R$. catawbiense. Data presented are mean $\pm \mathrm{SE}(\mathrm{n}=5$ independent measurements from three leaf discs per species; $2+2+1)$. Leaf samples were fixed and treated the same way as described in Fig. 3.

Epidermal cells have two features that are the key to preventing evaporative water loss: dense packing and coverage by cuticles. Cuticle wax in the abaxial and adaxial surfaces of the epidermis cells is considered to prevent water loss (Shepherd and Griffiths, 2006). Because rhododendron plants are shallow-rooted, they are particularly susceptible to leaf desiccation under windy and/or freezing conditions in winter. Our results showed that although the stomatal density of $R$. ponticum was much higher than that of $R$. catawbiense in both NA and CA plants (Fig. 6E), the "overall opening" of stomatal pores per unit leaf area (an integrated value of stomatal density and pore size) was much greater in $R$. catawbiense than that in
R. ponticum (Fig. 7G); it is noteworthy, however, that this conclusion has an underlying assumption that stomata have a similar degree of "openness" (or similar pore size) in NA and CA leaves. This feature indicates that the leaves of $R$. catawbiense may be more prone to winter desiccation than $R$. ponticum when soils are frozen, especially because this species frequently grows on open "balds." Thus, we suggest that accumulation of a relatively large amount of cuticle wax in the abaxial leaf surface (Figs. 6B and 6D) in R. catawbiense as well as thicker cuticles in the adaxial leaf surface of this species may help them reduce water loss in winter; a recent study of the relationship between leaf wax deposition and drought tolerance in tobacco demonstrated that leaf cuticular wax load increased significantly when plants were subjected to a periodic drought (Cameron et al., 2006).

We observed a reduction in stomatal density of the winter leaves relative to summer ones in the two Rhododendron species included in this study (Fig. 6E). This is a rather unexpected result because stomata are believed to be formed from initials early in leaf development and, therefore, stomatal density is not expected to change over time in mature, fully expanded leaves, the ones used here. Future studies focused at investigating temporal changes in the stomatal density (between seasons or even years because leaves last several years in this evergreen species) should provide further insight into this rather intriguing phenomenon.

In conclusion, the two species have evolved distinct leaf structural adaptations to winter conditions. The additional layer of upper epidermis and multiple palisade layers in $R$. ponticum leaves and thicker cuticle with wax and an extra palisade layer in $R$. catawbiense may constitute structural adaptations involved in reducing light stress during winter when photosynthesis is generally downregulated. Our results also indicated that whereas stomatal density of $R$. ponticum was higher than that of $R$. catawbiense leaves, the remarkably higher overall opening of stomatal pores per unit leaf area (an integrated value of stomatal density and pore size) in $R$. catawbiense might render this species more prone to winter desiccation and thus thermonasty may serve as a desiccationavoidance strategy in this species, perhaps in addition to serving a light-avoidance role. 


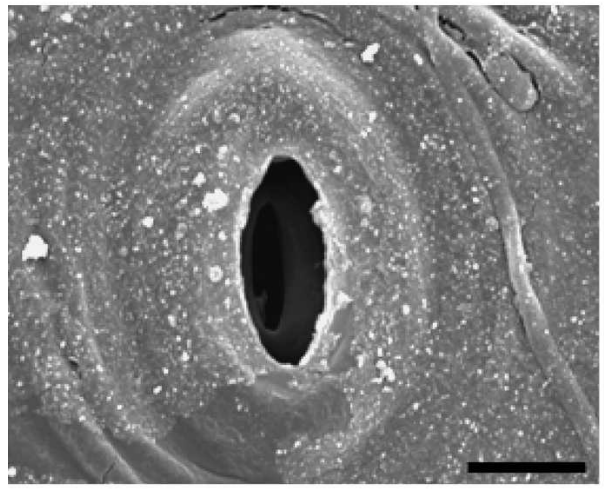

C
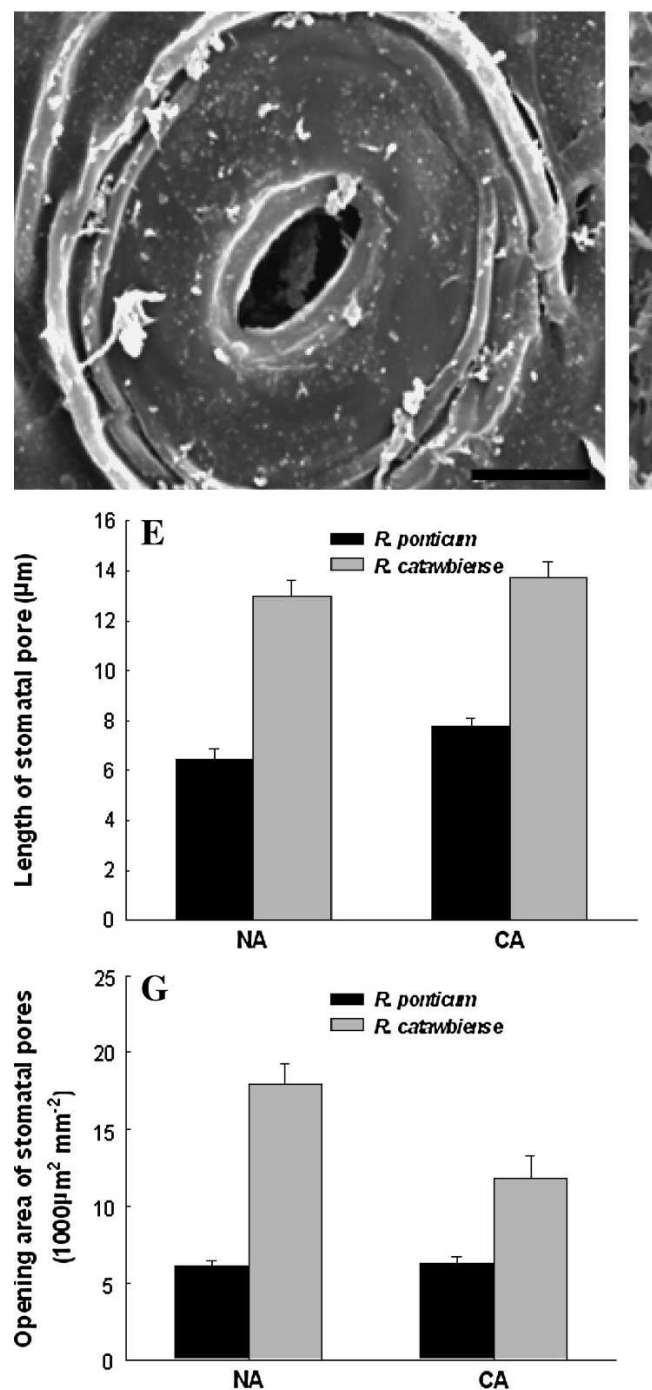

Fig. 7. Scanning electron micrographs showing the size $(\mu \mathrm{m})$ and opening area (an integrated value of stomatal density and pore size calculated as $\mu \mathrm{m}^{2} \cdot \mathrm{mm}^{-2}$ leaf area) of stomata pores of nonacclimated (NA) (A-B) and cold-acclimated (CA) (C-D) leaves from Rhododendron ponticum and $R$. catawbiense, respectively. $(\mathbf{A}, \mathbf{C})$ Images showing stomatal pores of NA and CA leaves from $R$. ponticum, respectively. Bars $=5 \mu \mathrm{m}$. (B, D) Images showing stomatal pores of NA and CA plants from $R$. catawbiense, respectively. Bars $=5 \mu \mathrm{m}$. $(\mathbf{E}, \mathbf{F}, \mathbf{G})$ Comparison of the length, width, and opening area of stomata pores from NA and CA leaves of $R$. ponticum and $R$. catawbiense, respectively. Data presented are mean $\pm \mathrm{SE}(\mathrm{n}=5$ independent measurements from three leaf discs per species; $2+2+1$ ). Leaf samples were fixed and treated the same way as described in Fig. 3.
B

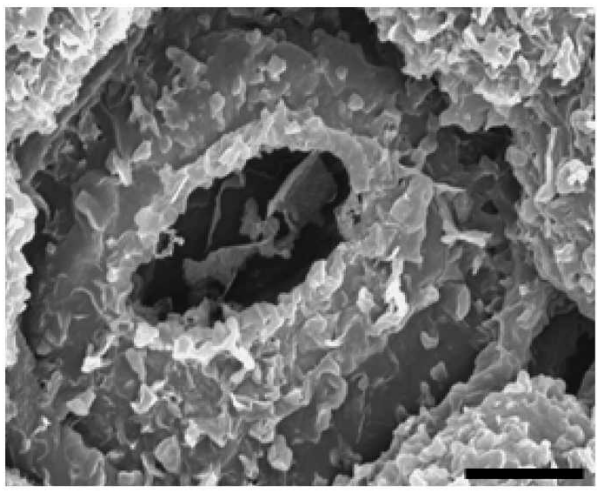

D
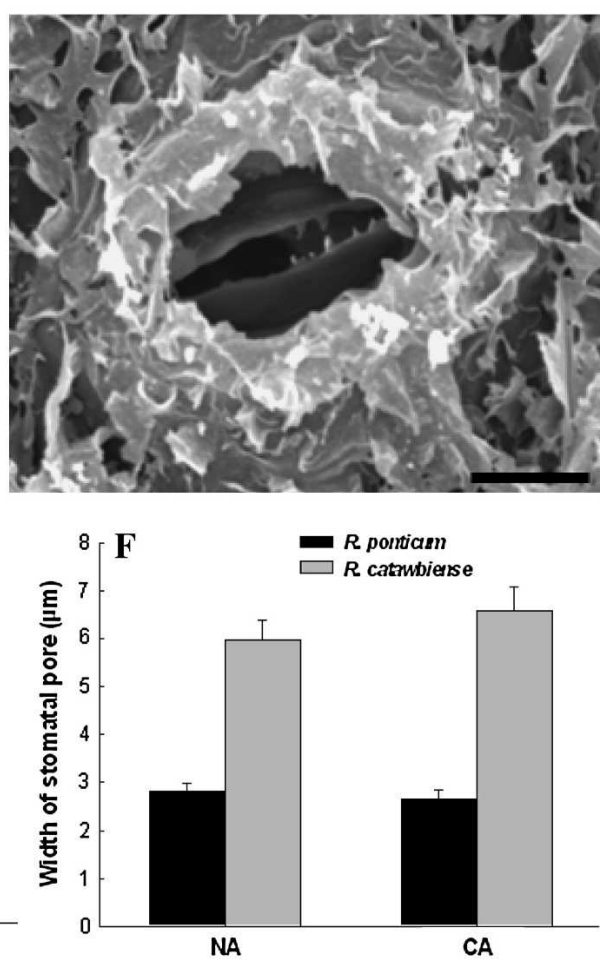

\section{Literature Cited}

Adams, W.W., III, C.R. Zarter, V. Ebbert, and B. Demmig-Adams. 2004. Photoprotective strategies of overwintering evergreens. Bioscience 54:41-49.

Anderson, J.M., W.S. Chow, and Y.I. Park. 1995. The grand design of photosynthesis: Acclimation of the photosynthetic apparatus to environmental cues. Photosynth. Res. 46:129-139.

Bao, Y. and E.T. Nilsen. 1988. The ecophysiological significance of leaf movements in Rhododendron maximum L. Ecology 69:1578-1587.

Björkman, O.B. 1981. Responses to different quantum flux densities, $p$. 57-107. In: Lange, O.L., P.S. Nobel, C.B. Osmond, and H. Ziegler (eds.). Encyclopedia of plant physiology, new series, Vol. 12 A. SpringerVerlag, Berlin, Germany.

Cameron, K.D., M.A. Teece, and L.B. Smart. 2006. Increased accumulation of cuticular wax and expression of lipid transfer protein in response to periodic drying events in leaves of tree tobacco. Plant Physiol. 140:176-183.

Chamberlain, D., R. Hyam, G. Argent, G. Fairweather, and K.S. Walter. 1996. The genus Rhododendron. Its classification and synonymy. Royal Botanic Garden, Edinburgh, Scotland.

Crombie, W.M.L. 1962. Thermonasty, p. 15-28. In: Ruhland, W. and E. Bünning (eds.). Encyclopedia of plant physiology, Vol. 17, Physiology of movements 2. Springer-Verlag, Berlin, Germany.

Endo, Y. and T. Miyauchi. 2006. Thermonasty of young main stems of Phryma leptostachya (Phrymaceae). J. Plant Res. 119:449-457.

Harris, G.C., V. Antoine, M. Chan, D. Nevidomskyte, and M. Königer. 2006. Seasonal changes in photosynthesis, protein composition and mineral content in Rhododendron leaves. Plant Sci. 170:314-325.

Heddad, M., H. Norén, V. Reiser, M. Dunaeva, B. Andersson, and I. Adamska. 2006. Differential expression and localization of early light-induced proteins in Arabidopsis. Plant Physiol. 142:75-87.

Huner, N.P.A., J.P. Palta, P.H. Li, and J.V. Carter. 1981. Anatomical changes in leaves of Puma rye in response to growth at cold-hardening temperatures. Bot. Gaz. 142:55-62.

Leach, D.G. 1961. Rhododendrons of the world and how to grow them. Scribner, New York, NY.

Liakoura, V., J.E. Bornman, and G. Karabourniotis. 2003. The ability of abaxial and adaxial epidermis of sun 
and shade leaves to attenuate UV-A and UV-B radiation in relation to the UV absorbing capacity of the whole leaf methanolic extracts. Physiol. Plant. 117:33-43.

Lichtenthaler, H.K., C. Buschmann, M. Döll, H.-J. Fietz, T. Bach, U. Kozel, D. Meier, and U. Rahmsdorf. 1981. Photosynthetic activity, chloroplast ultrastructure, and leaf characteristics of high-light and low-light plants and of 'sun' and 'shade' leaves. Photosynth. Res. 2:115-141.

Lim, C.C., R. Arora, and E.D. Townsend. 1998. Comparing Gompertz and Richards functions to estimate freezing injury in Rhododendron using electrolyte leakage. J. Amer. Soc. Hort. Sci. 123:246-252.

Martin, J.T. and B.E. Juniper. 1970. The cuticles of plants. Edward Arnold, London, UK.

Murchie, E.H. and P. Horton. 1997. Acclimation of photosynthesis to irradiance and spectral quality in British plant species: Chlorophyll content, photosynthetic capacity and habitat preference. Plant Cell Environ. 20:438-448.

Nilsen, E.T. 1987. Influence of water relations and temperature on leaf movements of Rhododendron species. Plant Physiol. 83:607-612.

Nilsen, E.T. 1992. Thermonastic leaf movements: A synthesis of research with Rhododendron. Bot. J. Linn. Soc. 110:205-233.

Nilsen, E.T. and A. Tolbert. 1993. Does winter leaf curling confer cold stress tolerance in Rhododendron? J. Amer. Rhododendron Soc. 47:98-104.

Niyogi, K.K. 1999. Photoprotection revisited: Genetic and molecular approaches. Annu. Rev. Plant Physiol. Plant Mol. Biol. 50:333-359.

Nobel, P.S. 1977. Internal leaf area and cellular $\mathrm{CO}_{2}$ resistance: Photosynthetic implications of variations with growth conditions and plant species. Physiol. Plant. 40:137-144.

Oguchi, R., K. Hikosaka, and T. Hirose. 2003. Does the change in light acclimation need leaf anatomy? Plant Cell Environ. 26:505-512.

Osborn, J.F. and T.N. Taylor. 1990. Morphological and ultrastructural studies of plant cuticular membranes. I. Sun and shade leaves of Quercus velutina (Fagaceae). Bot. Gaz. 151:465-476.

Palta, J.P. and P.H. Li. 1979. Frost-hardiness in relation to leaf anatomy and natural distribution of several Solanum species. Crop Sci. 19:665-671.

Peng, Y., W. Lin, H. Wei, S.L. Krebs, and R. Arora. 2008. Phylogenetic analysis and seasonal cold acclimation-associated expression of early light-induced protein genes of Rhododendron catawbiense. Physiol. Plant. 132:44-52.

Pino, M.T., J.S. Skinner, Z. Jeknic, P.M. Hayes, A.H. Soeldner, M.F. Thomashow, and T.H. Chen. 2008. Ectopic $A t C B F 1$ over-expression enhances freezing tolerance and induces cold acclimation-associated physiological modifications in potato. Plant Cell Environ. 31:393406.

Reed, D.W. and H.B. Tukey, Jr. 1982. Light intensity and temperature effects on epicuticular wax morphology and internal cuticle ultra- structure of carnation and brussels sprouts leaf cuticles. J. Amer. Hort. Sci. 107:417-420.

Sakai, A., L. Fuchigami, and C.J. Weiser. 1986. Cold hardiness in the genus Rhododendron. J. Amer. Soc. Hort. Sci. 111:273-280.

Sakai, A. and W. Larcher. 1987. Frost survival of plants: Responses and adaptation to freezing stress. Springer-Verlag, Berlin, Germany.

Shepherd, T. and D.W. Griffiths. 2006. The effects of stress on plant cuticular waxes. New Phytol. 171:469-499.

Shepherd, T., G.W. Robertson, D.W. Griffiths, A.N.E. Birch, and G. Duncan. 1995. Effects of environment on the composition of epicuticular wax from kale and swede. Phytochemistry 40:407417.

Sims, D.A. and R.W. Pearcy. 1992. Response of leaf anatomy and photosynthetic capacity in Alocasia macrorrhiza (Araceae) to a transfer from low to high light. Amer. J. Bot. 79:449-455.

Spurr, A.R. 1969. A low-viscosity epoxy resin embedding medium for electron microscopy. J. Ultrastruct. Res. 26:31-43.

Stefanowska, M., M. Kuraś, M. Kubacka-Zębalska, and A. Kacperska. 1999. Low temperature affects pattern of leaf growth and structure of cell walls in winter oilseed rape. Ann. Bot. (Lond.) 84:313-319.

Walters, R.G., F. Shephard, J.J.M. Rogers, S.A. Rolfe, and P. Horton. 2003. Identification of mutants of Arabidopsis defective in acclimation of photosynthesis to the light environment. Plant Physiol. 131:472-481.

Wei, H., A.L. Dhanaraj, L.J. Rowland, Y. Fu, S.L. Krebs, and R. Arora. 2005. Comparative analysis of expressed sequence tags (ESTs) from cold-acclimated and non-acclimated leaves of Rhododendron catawbiense Michx. Planta 221:406-416.

Weiser, C.J. 1970. Cold resistance and injury in woody plants. Science 169:1269-1278.

Wentworth, M., E.H. Murchie, J.E. Gray, D. Villegas, C. Pastenes, M. Pinto, and P. Horton. 2006. Differential adaptation of two varieties of common bean to abiotic stress-II. Acclimation of photosynthesis. J. Expt. Bot. 57:699-709.

Wild, A. and G. Wolf. 1980. The effect of different light intensities on the frequency and size of stomata, the size of cells, the number, size and chlorophyll content of chloroplasts in the mesophyll and the guard cells during the ontogeny of primary leaves of Sinapis alba. Zeits. Pflanzenphysiol. 97:325-342.

Zarter, C.R., B. Demmig-Adams, V. Ebbert, I. Adamska, and W.W. Adams, III. 2006a. Photosynthetic capacity and light harvesting efficiency during the winter-to-spring transition in subalpine conifers. New Phytol. 172:283-292.

Zarter, C.R., W.W. Adams, III, V. Ebbert, D.J. Cuthbertson, I. Adamska, and B. Demmig-Adams. 2006b. Winter down-regulation of intrinsic photosynthetic capacity coupled with up-regulation of Elip-like proteins and persistent energy dissipation in a subalpine forest. New Phytol. 172:272-282. 